Silva MDF, Bezerra CA, Alencar GOA, Barreto MFr,

PINTO NB, SOUTO RP, SARAIVA EMS
AVALIACÃO DA TERAPIA MEDICAMENTOSA DE PACIENTES PORTADORES DE OSTEOPOROSE, ATENDIDOS NA FARMÁCIA MUNICIPAL DO CRATO-CE

\title{
AVALIAÇÃO DA TERAPIA MEDICAMENTOSA DE PACIENTES PORTADORES DE OSTEOPOROSE, ATENDIDOS NA FARMÁCIA MUNICIPAL DE CRATO, CEARÁ
}

Evaluation of drug therapy of patients with osteoporosis, seen in the Pharmacy City of Crato, Ceará

Evaluación de la terapia farmacológica de los pacientes con osteoporosis, atendidos en la Farmacia Municipal de Crato, Ceará

Márcia Dayane de Freitas da Silva1', Camila Alencar Bezerra1, Gustavo de Oliveira Alencar ${ }^{1}$,

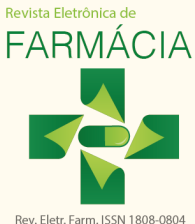
Maria de Fátima Rocha Barreto1, Natália Bitu Pinto², Ricardo Peres do Souto³, Emanuela Machado Silva Saraiva1*

${ }^{1}$ Faculdade de Juazeiro do Norte

2 Universidade Federal de Campina Grande

${ }^{3}$ Faculdade de Medicina do ABC

*E-mail: emanuelams@hotmail.com

\section{Resumo}

Introdução: A osteoporose é um distúrbio osteometabólico que se caracteriza pela redução da densidade mineral óssea, acarretando um aumento da fragilidade óssea, o que propicia um elevado risco de fraturas.

Objetivo: O objetivo desta pesquisa é avaliar os fatores relacionados ao tratamento medicamentoso de pacientes portadores de osteoporose atendidos na Farmácia Municipal da cidade de Crato, Ceará.

Metódos: Trata-se de um estudo do tipo exploratório descritivo com abordagem quantitativa, realizado em março de 2016.

Resultados: Dentre os entrevistados, 36,10\% afirmaram realizar tratamento da osteoporose a 3-4 anos e 37,82\% fazem acompanhamento médico anualmente. Os medicamentos destinados ao tratamento da osteoporose foram o alendronato de sódio $(69,75 \%)$ e carbonato de cálcio mais vitamina D3 (92,45\%). Durante a consulta médica, 97,48\% dos entrevistados afirmaram receber orientação sobre a posologia e 43,70\% sobre o horário de administração.

Conclusão: $O$ tratamento medicamentoso destinado aos portadores de osteoporose, no âmbito desta pesquisa, atendeu aos preceitos básicos que visam o controle da doença e a recuperação da saúde do paciente.

Palavras-chave: Osteoporose. Prescrição. Tratamento medicamentoso.

\section{Abstract}

Introduction: Osteoporosis is an osteometabolic disorder characterized by a reduction in bone mineral density, leading to an increase in bone fragility, which leads to a high risk of fractures.

Objective: The objective of this research was to evaluate the factors related to the drug treatment of patients with osteoporosis treated at the Municipal Pharmacy of the city of Crato, Ceará.

Methods: A descriptive exploratory study with a quantitative approach was carried out in March 2016.

Results: Among the interviewees, 36.10\% stated that they had osteoporosis treatment for 3-4 years, and 37.82\% had medical follow-up on an annual basis. Osteoporosis drugs were alendronate sodium $(69.75 \%)$ and calcium carbonate plus vitamin D3 (92.45\%). During the medical consultation, $97.48 \%$ of respondents stated that they received guidance on posology and $43.70 \%$ on the schedule of administration.

Conclusion: The medical treatment for osteoporosis patients, within the scope of this research, met the basic precepts that aim at the control of the disease and recovery of the patient's health.

Keywords: Osteoporosis. Prescription. Drug Treatment. 


\section{Resumen}

Introducción: La osteoporosis es un trastorno osteometabólico que se caracteriza por la reducción de la densidad mineral ósea, acarreando un aumento de la fragilidad ósea, lo que origina un alto riesgo de fracturas. Objetivo: El objetivo de esta investigación fue evaluar los factores relacionados al tratamiento medicamentoso de pacientes portadores de osteoporosis atendidos en la Farmacia Municipal de la ciudad de Crato, Ceará.

Métodos: Se trató de un estudio del tipo exploratorio descriptivo de enfoque cuantitativo, realizado en marzo de 2016.

Resultados: Entre los entrevistados, el 36,10\% afirmaron realizar tratamiento de la osteoporosis hace 3 o 4 años y el 37,82\% tiene atendimiento médico anualmente. Los medicamentos destinados al tratamiento de la osteoporosis fueron el alendronato de sodio $(69,75 \%)$ y el carbonato de calcio, además de la vitamina D3 (92,45\%). Durante la consulta médica, el 97,48\% de los entrevistados afirmaron recibir orientación sobre la posología y el 43,70\% sobre el horario de administración.

Conclusion: El tratamiento medicamentoso destinado a los portadores de osteoporosis, en el marco de esta investigación, atendió a los preceptos básicos que se dirigen al control de la enfermedad y la recuperación de la salud del paciente.

Palabras clave: La osteoporosis. , Prescripción. Tratamiento medicamentoso.

\section{INTRODUÇÃO}

A osteoporose é um distúrbio osteometabólico que se caracteriza pela redução da densidade mineral óssea, no qual gera um aumento da fragilidade óssea e, consequentemente, o risco de fraturas. É uma doença multifatorial, sendo mais prevalente em idosos, influenciada tanto por fatores genéticos quanto ambientais ${ }^{(1)}$.

No mundo todo, a osteoporose acomete $7 \%$ de homens e $17 \%$ de mulheres, dessas, 70\% estão na faixa etária igual ou superior a 80 anos. Nos Estados Unidos, Europa e Japão, aproximadamente 75 milhões de pessoas são acometidas por essa doença e estima-se que poderão ocorrer mais de oito milhões de fraturas de quadril nos próximos 50 anos, já na América Latina, estudos relataram que a frequência é de dois casos a cada dez mil pessoas ${ }^{(2)}$.

Dentre os fatores de risco associados à osteoporose podem ser citados: idade avançada, sexo feminino, raça caucasoide, histórico familiar, desordens osteometabólicas, ingestão baixa de cálcio e vitamina D, sedentarismo, artrose, consumo de bebidas alcoólicas e tabagismo ${ }^{(1,3)}$.

A osteoporose é uma das principais causas de morbidade e mortalidade em idosos, com diversas complicações como dor crônica, depressão, deformidades ósseas e perda da independência. A dificuldade e o alto custo do tratamento para o Sistema de Saúde tornam pertinente o desenvolvimento de métodos capazes de identificar o grupo de maior risco para que sejam implantadas medidas preventivas de fraturas osteoporóticas ${ }^{(4)}$.

O tratamento da osteoporose consiste em medidas não medicamentosas e medicamentosas. No primeiro, deve ser recomendada a atividade física para redução do risco de fraturas, com uma avaliação prévia de um profissional na prevenção de quedas; evitar tabagismo e álcool. No segundo, a maior parte dos estudos que demonstrou eficácia na prevenção de fraturas foi realizada em populações de pacientes com osteoporose na pós-menopausa, sendo que as evidências dizem respeito à prevenção de fraturas osteoporóticas induzidas por glicocorticoides; na osteoporose masculina as evidências foram menos robustas. Nos pacientes com alto risco de fraturas osteoporóticas, o medicamento a ser utilizado deve ser avaliado considerando os benefícios e riscos potenciais do tratamento(4).

A ocorrência de fratura osteoporótica durante o tratamento não significa necessariamente que foi falho. As evidências não existem, mas têm mostrado bons resultados, trazendo benefícios de densitometrias seriadas para avaliação de resposta ou definição de conduta após o início do tratamento. Após completar o tempo de tratamento proposto, os pacientes devem ter o acompanhamento mantido, com avaliações constando anamnese e exame físico, a cada 6 a 12 meses ${ }^{(5)}$. 
O tratamento da osteoporose se torna, às vezes, difícil, por ter alto custo e o trato das fraturas osteoporóticas ser extremamente demorado. Além de tudo, há pouca orientação em relação à doença e seu tratamento, tornando assim um dos motivos a dificultar a adesão ao tratamento.

Diante desse contexto, o objetivo da presente pesquisa é avaliar os fatores relacionados ao tratamento medicamentoso dos pacientes portadores de osteoporose, atendidos na Farmácia Central da Rede Municipal de Saúde de Crato, Ceará.

\section{MATERIAL E MÉTODOS}

O estudo caracteriza-se como exploratório descritivo com abordagem quantitativa, de delineamento transversal e prospectivo, com entrevista de 119 portadores de osteoporose na Farmácia Municipal da cidade de Crato-CE, em março de 2016. Foi aplicado um questionário que abordava aspectos relacionados à idade e sexo do paciente, tempo de tratamento do quadro clínico de osteoporose, existência de copatologia, medicamentos em uso, frequência do acompanhamento médico e o tipo de orientação recebida durante a prescrição do medicamento. Avaliou-se também a prescrição apresentada pelo entrevistado, no intuito de observar informações registradas no receituário.

Os critérios de inclusão para participar da pesquisa foram pacientes com diagnóstico médico de osteoporose, com idade igual ou superior a 30 anos, de ambos os sexos e que aceitaram participar voluntariamente, tendo em mãos receita médica com até 90 dias da data da emissão.

A presente pesquisa obedeceu aos preceitos éticos, conforme a Resolução CNS/MS N466, de 12/2012, que trata de pesquisa envolvendo humanos, os quais determinam a garantia do anonimato da identidade do participante da pesquisa mesmo na divulgação dos dados obtidos( ${ }^{(6)}$. Bem como foi submetida à apreciação pelo Comitê de Ética em Pesquisa da Faculdade de Juazeiro do Norte - FJN e aprovada sob número CAAE 50663215.5.0000.5624.

\section{RESULTADOS E DISCUSSÃO}

Foram realizadas 119 entrevistas com pacientes portadores de osteoporose atendidos na Farmácia Central da cidade de Crato-CE. Dentre os entrevistados, houve prevalência do sexo feminino da ordem de 113 mulheres (95\%) e da faixa etária entre 56 a 65 anos (38,65\%). A Figura 1 apresenta a distribuição dos pacientes entrevistados, de acordo com o gênero e faixa etária.

Figura 1. Distribuição dos pacientes portadores de osteoporose, por gênero e faixa etária. Crato-CE, 2016.

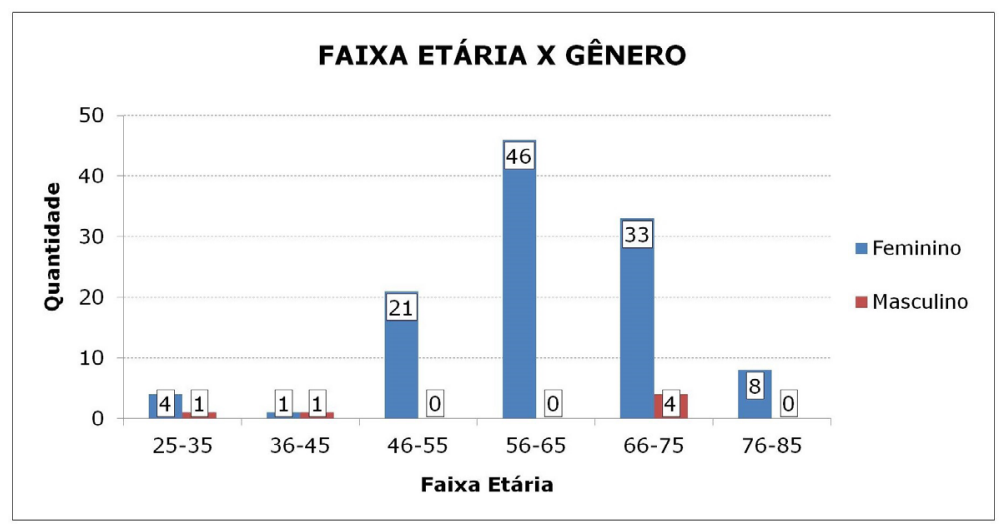


A osteoporose atinge, principalmente, pessoas idosas e tem uma incidência maior nas mulheres, sobretudo após a menopausa, quando ocorre uma diminuição dos níveis do hormônio estrogênio circulante, o que leva a uma ativação nos ciclos de remodelação óssea, com predomínio nas fases de reabsorção em relação à formação, com consequente diminuição da massa óssea ${ }^{(3,7)}$.

O equilíbrio entre formação e reabsorção óssea existe em ambos os sexos, porém com o avançar da idade se torna progressivamente negativo e a maioria dessa perda ocorre após os 65 anos. Os homens são menos suscetíveis a desenvolver osteoporose do que as mulheres por ganharem mais massa óssea durante a puberdade e a perda é durante o envelhecimento, tudo isso porque os homens não têm uma redução rápida de estrógenos como as mulheres ${ }^{(8)}$.

Os estudos estimam que a partir dos 40 anos pode ocorrer perda de cerca de $5 \%$ de massa muscular e o mesmo vale com o passar de cada década ${ }^{(9)}$. A taxa de perda óssea em mulheres com hipoestrogenismo na pós-menopausa é de 0,5-1,5\% por ano, com uma pequena porcentagem dessas, são "perdedoras rápidas de osso" que podem chegar a perder de $3 \%$ a $5 \%$ de massa óssea por ano(8).

Oliveira et al. (2010) relatam que a osteoporose primária no homem tende a ocorrer acima dos 70 anos de idade, decorrente de uma combinação de fatores, que associam diminuição da absorção de cálcio intestinal, ativação reduzida da vitamina $\mathrm{D}$, declínio do tempo de vida dos osteoblastos e diminuição da concentração dos hormônios sexuais ${ }^{(10)}$.

Destaca-se que a população do presente estudo busca o serviço de saúde em idade avançada, o que poderia justificar a maior gravidade das lesões encontradas nos pacientes portadores de osteoporose. Em conjunto, esses resultados podem sugerir que esses indivíduos procuram menos atendimento profissional ou que a investigação da massa óssea ocorre com menor frequência.

Ao avaliarmos a distribuição dos pacientes de acordo com o tempo de tratamento da osteoporose, verificamos que a maioria $(36,10 \%)$ declara realizar terapia entre 03-04 anos, conforme demonstrado pela Tabela 1.

Tabela 1. Distribuição dos pacientes por tempo de tratamento para a osteoporose. Crato-CE, 2016.

\begin{tabular}{cccc}
\hline Anos & $\mathbf{n}$ & $\mathbf{\%}$ \\
\hline $1-2$ & 25 & $21,00 \%$ \\
$3-4$ & 43 & $36,10 \%$ \\
$5-6$ & 29 & $24,40 \%$ \\
$8-9$ & 5 & $4,20 \%$ \\
10 & 11 & $9,25 \%$ \\
$>10$ & 6 & $5,05 \%$ \\
\hline Total & $\mathbf{1 1 9}$ & $\mathbf{1 0 0} \%$ \\
\hline
\end{tabular}

Fonte: Elaborado pelos autores (2016).

Segundo o Ministério da Saúde (2014), através do Protocolo Clínico e Diretrizes Terapêuticas da Osteoporose, a maioria dos estudos embasa o uso de medicamentos na prevenção de fraturas osteoporóticas com seguimento de 03-05 anos, o tratamento com alendronato por cinco anos associa-se à redução de fraturas. Portanto, o tratamento da osteoporose deve ser mantido por cinco anos, porém não existe um consenso de quanto tempo deve dar continuidade a essa terapia. Entretanto, 
em mulheres que não apresentarem alto risco de fraturas deve ser interrompido após cinco anos. Para pacientes em uso de alendronato com piora da massa óssea pode ser estendido por mais tempo, porém não há evidências clínico-epidemiológicas consistentes nesse sentido, sendo obrigatória a individualização da condutat ${ }^{(11,12)}$.

Outro dado investigado na pesquisa foi o relato de outras patologias pelos pacientes entrevistados, no qual se verificou que 96 deles (80,68\%) apresentavam outras patologias além da osteoporose, as quais foram distribuídas na Figura 2.

Os resultados obtidos demonstram que além da osteoporose, os entrevistados apresentam outras doenças crônicas não transmissíveis, com destaque a hipertensão arterial que foi declarada por 57 (47,90\%) pacientes, seguida da artrose, presente em 31 (26,05\%) entrevistados.

Figura 2. Copatologias declaradas por pacientes portadores de osteoporose. Crato-CE, 2016

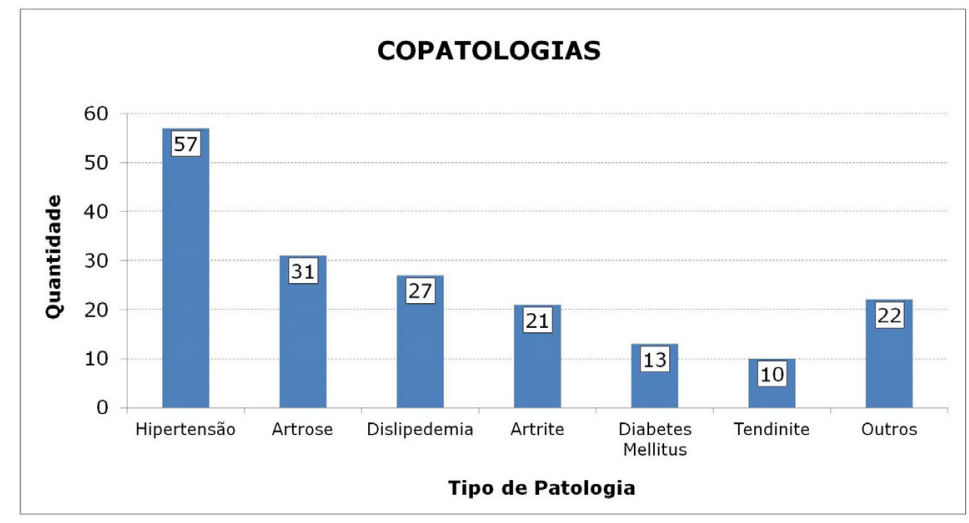

A hipertensão arterial sistêmica (HAS) é considerada um dos principais fatores de risco, sendo a mais importante em relação aos problemas de saúde pública no Brasil e no mundo. Trata-se de condição clínica multifatorial caracterizada pela elevação dos níveis da pressão arterial (PA) acima de $140 \mathrm{mmhg}$ na pressão arterial sistólica (PAS) e 90mmhg na diastólica (PAD), no qual possui alta prevalência e baixas taxas de controle. Nos últimos 10 anos, o fator de risco precursor e potencial das doenças apontadas foi as cardiovasculares (DCV) que representam 30\% dos casos que acometem aproximadamente 17 milhões de pessoas, geram causas de morte e até $50 \%$ da mortalidade do conjunto das Doenças Crônicas Não Transmissíveis $(\mathrm{DCNT})^{(13)}$.

A segunda doença mais citada foi a artrose, que se trata de uma enfermidade articular degenerativa, caracterizada, geralmente, por uma destruição lenta e progressiva da cartilagem, com causa desconhecida ${ }^{(14)}$.

Embora osteoartrose e osteoporose sejam doenças relacionadas a disfunções esqueléticas, inquéritos epidemiológicos, raramente, associam ambas as doenças. Porém, a presença de uma pode ser considerada fator de proteção à outra, pois a conformidade óssea aumentada na osteoporose mantém a cartilagem articular preservada. Existem poucos relatórios sobre osteoartrose na fase inicial, no entanto, investigações recentes têm relatado graves alterações microscópicas no osso da cartilagem em estágios avançados na osteoartrose, como baixa mineralização óssea e rigidez mecânica, bem como deteriorações consideráveis na cartilagem articular, sugerindo desenvolvimento de osteoartrose em pacientes com osteoporose e que o tratamento da osteoporose pode ajudar a prevenir a progressão de osteoartrose $\mathrm{e}^{(14)}$. 
Com relação aos medicamentos mais utilizados para o tratamento da osteoporose foi possível verificar o predomínio de prescrições de Carbonato de cálcio com adição de vitamina D (92,45\%), seguido do Alendronato de sódio (69,75\%), uma vez que são disponibilizados à população através da rede de atenção básica à saúde. A Tabela 2 relaciona os medicamentos destinados ao tratamento da osteoporose identificados na presente pesquisa.

Tabela 2. Medicamentos prescritos para o tratamento da osteoporose. Crato-CE, 2016.

\begin{tabular}{ccc}
\hline Medicamento & Quantidade & $\%$ \\
\hline Carbonato de cálcio + D3 & 110 & $92,45 \%$ \\
Alendronato de sódio & 83 & $69,75 \%$ \\
Calcitriol & 05 & $4,20 \%$ \\
Raloxifeno & 03 & $2,52 \%$ \\
Risedronato & 02 & $1,68 \%$ \\
\hline
\end{tabular}

Fonte: Elaborado pelos autores (2016).

O início do tratamento da osteoporose deve ser realizado através da suplementação de cálcio e vitamina $D$, utilizando-se diferentes sais de cálcio, sendo o mais indicado o carbonato de cálcio, pois apresenta cerca de $40 \%$ de cálcio elementar ${ }^{(15)}$.

A suplementação de vitamina $\mathrm{D}$ ajuda na recuperação da deficiência da densidade mineral óssea, além disso, reduz o risco de quedas e fraturas, pois tem a função de regular o metabolismo de cálcio/fósforo, garantindo assim a saúde dos ossos ${ }^{(15)}$. Diante disso, vale resaltar que o tratamento isolado pode aumentar o risco de hipercalcemia e hipercalciúria, já os bifosfonatos segundo os estudos mostraram podem causar hipocalcemia. Portanto, sugere-se que uma terapia combinando tais agentes possa ser bastante efetiva para melhorar a homeostase do cálcio e a massa óssea em pacientes portadores de osteoporose ${ }^{(17)}$.

Entre os medicamentos que reduzem fraturas osteoporóticas, a classe dos bifosfonatos é a que contém mais informações em termos de efetividade e segurança, sendo a primeira linha de tratamento da osteoporose, com estudos de até dez anos de seguimento publicados. A comparação do bifosfonatos entre o uso diário e semanal mostram maiores taxas de adesão e persistência de tratamento em favor do uso semana|(5,12).

O alendronato é indicado para prevenir fraturas primárias em pacientes portadores de osteoporose, havendo evidência de sua efetividade na redução de incidência de fraturas vertebrais, não vertebrais e do quadri|(5). O risedronato é um potente bifosfonato que aumenta a densidade mineral óssea e produz rápida redução de fraturas em mulheres com osteoporose após a menopausa, em combinação com a terapia de reposição hormonal(18). Estudos científicos mostram que o uso do risedronato leva a diminuição do risco de ocorrência de fraturas vertebrais e não vertebrais em $41 \%$ e 39\%, respectivamente, sendo efetivo e bem tolerado por mais de sete anos. A escolha de alendronato de sódio ou risedronato de sódio como representantes da classe baseia-se na maior experiência de seu uso e no menor custo(11,12).

As principais ações do calcitriol são a estimulação da absorção do $\mathrm{Ca}^{2+}$ e do fosfato no intestino e a mobilização de $\mathrm{Ca}^{2+}$ a partir do osso, mas ele também aumenta a reabsorção de $\mathrm{Ca}^{2+}$ nos túbulos renais. Seus efeitos nos ossos envolvem a maturação dos osteoclastos e a estimulação indireta da atividade deles ${ }^{(19)}$ 
O calcitriol em concentrações fisiológicas promove a mobilização do cálcio para o osso, ao passo que a administração de grandes doses gera remodelação óssea excessiva ${ }^{(20)}$. A reposição de vitamina $D$ é realizada por meio da administração da vitamina (colecalciferol) ou de seus análogos, como o calcitriol. Esse é um metabolito ativo da vitamina D, não é depositado no organismo, o que explica sua meia vida curta, quando comparado ao colecalciferol(12).

O raloxifeno é o modulador seletivo do receptor de estrógeno (SERM) mais usado para prevenção e tratamento da osteoporose e a avaliação do seu uso de um período de três anos entre as mulheres mostraram que houve aumento da densidade mineral óssea em coluna vertebral e colo do fêmur, reduzindo assim o risco de fratura vertebral(21).

Nos pacientes com distúrbio de deglutição, com intolerância ou falha terapêutica dos tratamentos de primeira linha (bifosfonatos), a utilização de raloxifeno ou estrógenos conjugados deve ser considerada ${ }^{(12)}$.

Outro dado levantado pela pesquisa foi o consumo de medicamentos pelo entrevistado além daqueles destinados ao tratamento da osteoporose. Em consonância com a população de estudo, a classe terapêutica mais relatada pelos entrevistados foram os analgésicos (96,63\%), conforme apresentado na Tabela 3.

Tabela 3. Prevalência dos medicamentos utilizados pelo paciente portador de osteoporose. Crato-CE, 2016.

\begin{tabular}{ccc}
\hline Classe Terapêutica & Quantidade & $\%$ \\
\hline Analgésico & 115 & $96,63 \%$ \\
Anti-hipertensivo & 75 & $63,02 \%$ \\
Glicocorticoides & 54 & $45,38 \%$ \\
Hipolipêmicos & 21 & $17,65 \%$ \\
Hipoglicemiantes & 12 & $10,08 \%$ \\
Outros & 45 & $37,81 \%$ \\
\hline
\end{tabular}

Fonte: Elaborado pelos autores (2016).

Nos indivíduos com osteoporose as dores vêm das consequências ósseas, como a diminuição da espessura ou da densidade e não da doença em si, uma vez que esta rarefação leva à perda do trabeculado ósseo, podendo resultar em fraturas. A dor também acomete na postura, sendo crônica, resultando em microfraturas vertebrais, levando a deformidades posturais(22).

Para o tratamento da dor aguda, na qual apresenta episódios de fratura vertebral compressiva, é indicado inicialmente o repouso no leito, limitado de uma ou duas semanas no máximo, associado a um posicionamento adequado, promovendo relativa neutralidade na coluna e na pelve. Meios físicos também são muito úteis como o calor superficial, a massoterapia (para o espasmo muscular) e a estimulação elétrica transcutânea (TENS). Se persistirem as dores, é indicada a utilização da órteses vertebrais a fim de melhorar a postura, com isso, as dores são aliviadas e as fraturas vertebrais compressivas estabilizadas. A reabilitação na osteoporose, além de aliviar as dores, preserva a integridade do esqueleto e melhora a mobilidade funcional(23).

A segunda classe de medicamentos mais citada pelos entrevistados foram os anti-hipertensivos (63,02\%). Segundo estudos, o uso de bloqueadores de receptor de angiotensina e os diuréticos tiazídicos estiveram associados a um menor risco de fraturas, principalmente no fêmur, quando comparados ao uso de bloqueadores dos canais de cálcio ${ }^{(24)}$. 
O uso de glicocorticoides $(45,38 \%)$ apresenta-se como a terceira classe terapêutica mais utilizada pelos pacientes entrevistados. Essa classe de medicamentos é muito prescrita para inflamação, principalmente em casos de osteoartrite, artrite reumatoide e doenças autoimunes, mas a prescrição deve ser previamente avaliada, pois possuem severos efeitos adversos sendo muito deles não desejáveis(25).

Esses esteroides possuem efeito catabólico sobre o músculo e sobre a vitamina $D$, que compromete a mineralização óssea. Com a diminuição da absorção intestinal de cálcio, tem o aumento na eliminação do cálcio urinário, redução na secreção do hormônio do crescimento $(\mathrm{GH})$ e as alterações no metabolismo dos esteroides sexuais e da pulsatilidade do hormônio da paratireoide (PTH). Tudo isso apresenta efeitos negativos indiretos dos glicocorticoides na saúde óssea. O próprio tecido ósseo é alvo para os glicocorticoides, as pesquisas têm evidenciado que os esteroides possuem ação direta sobre as células ósseas tendo como principal mecanismo na gênese da osteoporose resultante ${ }^{(26)}$.

A osteoporose glicocorticoide-induzida deve ser especialmente considerada, porque pode levar a uma perda óssea importante e a fraturas ósseas, nas doses usuais (2,5 a 7,5 mg de prednisona ao dia), em relativamente curto período de uso de três a seis meses ${ }^{(5)}$.

Estudos apontam que, aproximadamente, $30 \%$ a $50 \%$ das pessoas que usam glicocorticoides por mais de seis meses apresentam algum tipo de fratura, decorrente do efeito maléfico sobre o osso. Sendo este efeito mais comum entre mulheres na pós-menopausa e em crianças (27).

Foi questionada na pesquisa a frequência com que o paciente realiza acompanhamento médico relacionado à osteoporose, $34(28,58 \%)$ afirmaram realizar acompanhamento a cada três meses, $33(27,74 \%)$ a cada seis meses, $45(37,82 \%)$ a cada um ano e 07 (5,86\%) pacientes relataram realizar acompanhamento médico em um período superior a um ano (Figura 3).

Figura 3. Frequência da realização do acompanhamento médico pelo portador de osteoporose. Crato-CE, 2016.

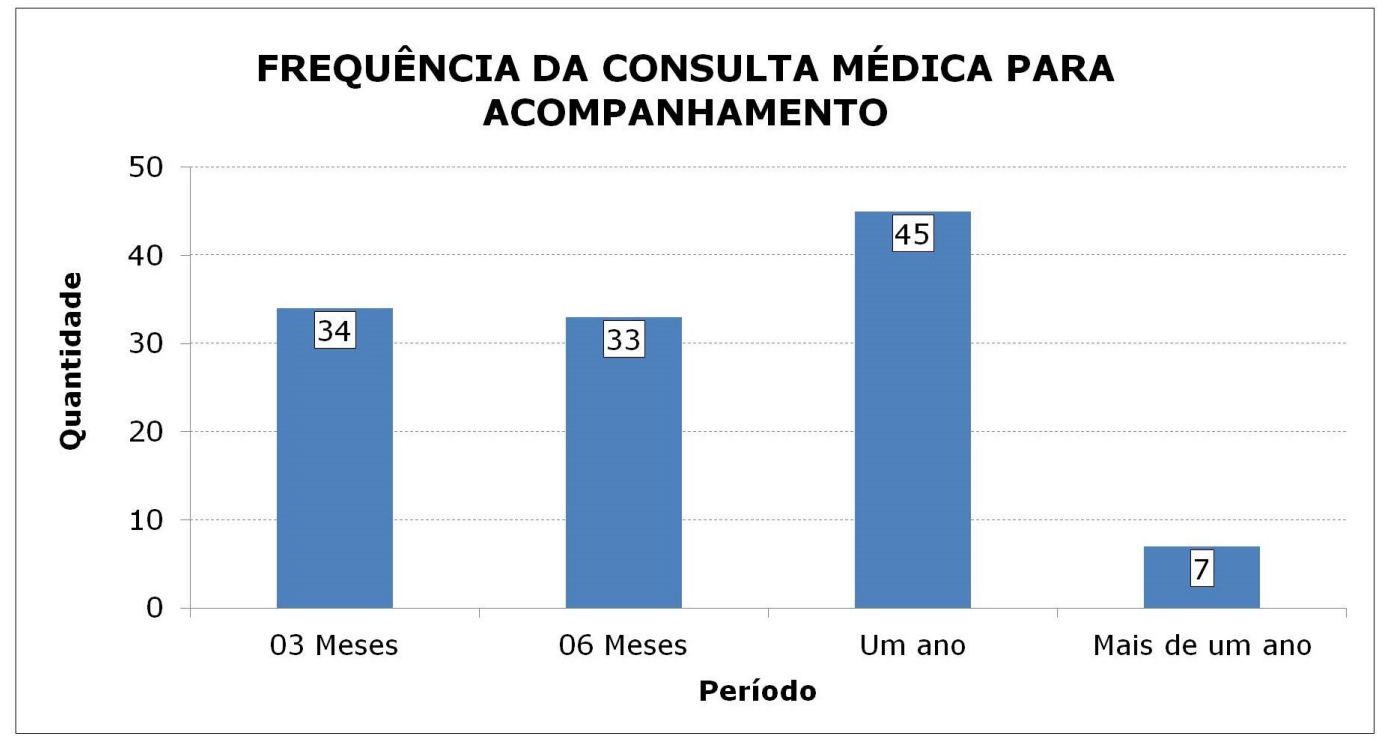


Quanto ao recebimento de orientação no ato da prescrição médica sobre a terapia medicamentosa da osteoporose, averiguamos que 64 (54\%) entrevistados recebem algum tipo de orientação e 55 (46\%) não recebe nenhuma orientação a respeito.

Dentre os entrevistados que afirmaram ter recebido orientação médica sobre a terapia medicamentosa, 63 (52,95\%) pacientes citaram que no ato da consulta receberam orientação sobre a posologia, 57 (47,90\%) mencionaram horário de administração, 11 (9,25\%) relataram que o médico forneceu orientação sobre a finalidade terapêutica do medicamento e 10 $(8,40 \%)$ receberam orientação sobre a importância da alimentação, como mostra a Tabela 4.

Tabela 4. Informações fornecidas pelo prescritor ao portador de osteoporose. Crato-CE. 2016.

\begin{tabular}{ccc}
\hline Orientações recebidas & Quantidade & $\%$ \\
\hline Posologia & 63 & $52,95 \%$ \\
Horário de administração & 57 & $47,90 \%$ \\
Finalidade terapêutica & 11 & $9,25 \%$ \\
Alimentação & 10 & $8,40 \%$ \\
\hline
\end{tabular}

Fonte: Elaborado pelos autores (2016).

Assugeni et al. (2007) destacam que todo paciente deve estar corretamente informado sobre seu diagnóstico, prognóstico e riscos em cada etapa do tratamento. Portanto, uma informação correta ao paciente em linguagem acessível é um dever do médico e representa uma profilaxia de complicações legais(28).

Camargo et al. (2007) relatam que se faz necessário repassar as recomendações essenciais para administração correta do medicamento, além do papel fundamental da comunicação dos clínicos com seus pacientes para o sucesso do tratamento, sendo necessário o aprimoramento na transmissão das informações adequadas ao paciente ${ }^{(29)}$.

Quando avaliamos a receita médica do paciente com relação à prescrição em Denominação Comum Brasileira (DCB), apenas 83 prescrições (70\%) estavam em DCB e 36 (30\%) estavam com o nome comercial do medicamento.

A Organização Mundial da Saúde (OMS) considera que a prescrição por nome genérico é um dos indicadores para avaliar a qualidade de medicamentos, pois promove o uso racional, no qual reduzem os custos destes e, consequente, melhoria do acesso, da adesão, do tratamento e diminuição até mesmo dos erros de medicação, portanto são adotadas as estratégias em Denominação Comum Brasileira (DCB)(30).

Santos et al. (2004) relatam nas análises das prescrições apenas que 30,6\% delas era pelo nome genérico, oriundas do SUS, sendo considerados baixos os dados, já que neste âmbito a prescrição médica deve, obrigatoriamente, ser realizada pela denominação genérica. No entanto, o resultado da presente pesquisa mostra que as prescrições em DCB ocorreram em $70 \%$ das receitas analisadas ${ }^{(31)}$.

As legislações brasileiras de saúde exigem o uso da nomenclatura genérica, quando a prescrição ocorrer em âmbito público de saúde. O uso do nome de referência do medicamento pode ser influenciado pela inexistência do mesmo nas listas de medicamentos essenciais; pela falta de conhecimento sobre tais listas e a legislação atual, bem como pelas estratégias de marketing na indústria farmacêutica, enfatizando a marca dos produtos ${ }^{(32)}$. 
Com base nos dados coletados, foi possível avaliar e classificar as informações presentes nas prescrições, como mostra a Tabela 5, que aponta predominância maior para posologia com 116 receitas $(97,48 \%)$ apresentando quantidade e dose para o tratamento. Com relação à frequência, essa informação estava contida em apenas 35 (29,42\%). Outro dado significativo encontrado no estudo foi em relação ao horário de administração, apenas $52(43,70 \%)$ apresentavam a informação e somente $17(14,28 \%)$ indicavam o tempo de tratamento que o paciente deveria realizar com o determinado medicamento.

Tabela 5. Prevalência das informações registradas na prescrição médica destinada ao paciente portador de osteoporose. Crato-CE, 2016.

\begin{tabular}{ccccc}
\hline Itens & Sim & $\%$ & Não & $\%$ \\
\hline Posologia & 116 & $97,48 \%$ & 3 & $2,52 \%$ \\
Horário de administração & 52 & $43,70 \%$ & 67 & $56,30 \%$ \\
Frequência & 35 & $29,42 \%$ & 84 & $70,58 \%$ \\
Tempo de tratamento & 17 & $14,28 \%$ & 102 & $85,72 \%$ \\
\hline
\end{tabular}

Fonte: Elaborado pelos autores (2016).

A prescrição de medicamentos é um documento de valor legal pelo qual se responsabilizam, perante o paciente e sociedade, aqueles que prescrevem, dispensam e administram os medicamentos. É regida por certos preceitos gerais, de forma a não deixar dúvida nem tão pouco dificuldade de realizar a interpretação(33).

Conforme o Manual Prático para Prescrição de Medicamentos, na prescrição deve constar as seguintes informações: Nome, forma farmacêutica; a quantidade total de medicamento (número de comprimidos, drágeas, ampolas, envelopes), de acordo com a dose e a duração do tratamento; a via de administração, o intervalo entre as doses, a dose máxima por dia e a duração do tratamento; nome, endereço e telefone do prescritor, de forma a possibilitar contato em caso de dúvidas ou ocorrência de problemas relacionados ao uso de medicamentos prescritos, e data ${ }^{(33)}$.

Madruga (2011) relata que a transparência da informação, a compreensão da receita médica e o conhecimento da prescrição proporcionam uma boa adesão ao tratamento. Sendo atribuição do prescritor esclarecer e tirar todas as dúvidas do paciente, bem como se colocar à disposição para possíveis efeitos adversos ${ }^{(34)}$.

\section{CONCLUSÃO}

Os pacientes entrevistados são em maior parte do gênero feminino, encontram-se a maioria na faixa etária de 56 a 65 anos de idade, apresentam outras patologias sendo a hipertensão a mais mencionada.

A maioria dos entrevistados realizam tratamento da osteoporose em média a três, quatro anos, faz acompanhamento médico anualmente, bem como necessita de associação de medicamentos para tratar a osteoporose, tal como a associação de alendronato de sódio e carbonato de cálcio adicionado de vitamina $\mathrm{D}$, tratamento básico para osteoporose.

Acredita-se que devido às dores crônicas apresentadas pela maioria dos entrevistados, o consumo elevado de medicamentos analgésicos foi justificável. 
Por meio da análise do receituário dos entrevistados, percebeu-se que a maioria destes foi prescrita utilizandose a Denominação Comum Brasileira, fato que atende os preceitos do SUS, além de possibilitar a intercambiabilidade do medicamento prescrito.

Durante a consulta médica, a maioria dos entrevistados afirmou receber orientação relacionada à posologia e ao horário de administração do medicamento destinado ao tratamento da osteoporose.

No entanto, propõe-se que os profissionais da saúde, não só o prescritor, sejam preparados para orientar o paciente em relação à terapia medicamentosa, como também as formas de prevenção da osteoporose. Umas das ferramentas para prevenção da osteoporose é a educação em saúde voltada para a adoção de hábitos saudáveis de vida e alimentação, assim como o uso racional de medicamentos que possibilitem a diminuição da massa óssea.

\section{REFERÊNCIAS}

1. Camargos MCS, Bomfim WC. Osteoporose e Expectativa de Vida Saudável: estimativas para o Brasil em 2008. Rio de Janeiro. Cad. saúde colet., 2017; 25(1): 106-112. DOI: http://dx.doi.org/10.1590/1414-462X201700010150.

2. Moraes LFS, Silva EN, Silva DAS, Paula AP. Gastos com o tratamento da osteoporose em idosos do Brasil (2008 - 2010): análise dos fatores associados. São Paulo. Rev. bras. epidemiol., 2014; 17(3): 719-734. DOI: http://dx.doi.org/10.1590/18094503201400030012.

3. Rodrigues IG, Barros MBA. Osteoporose autorreferida em população idosa: pesquisa de base populacional no município de Campinas, São Paulo. São Paulo. Rev. bras. epidemiol., 2016, 19(2): 294-306. DOI: http://dx.doi.org/10.1590/19805497201600020007.

4. Ministério da Saúde (BR). Portaria No 224, de 26 de Março de 2014. Aprova o Protocolo Clínico e Diretrizes Terapêuticas da Osteoporose. Disponível em: http://www.poderesaude.com.br/novosite/images/ Publica\%C3\%A7\%C3\%B5es_27.03.2014_-_Il.pdf.

5. Brasil. Prefeitura Municipal de São Bernardo do Campo. Secretaria de Saúde. Coordenação do Departamento de Apoio a Gestão do SUS. Protocolo clínico e diretrizes na prevenção e tratamento da osteoporose - Exames de Apoio Diagnóstico: endoscopia digestiva alta e colonoscopia. Vol. 01 - $1^{\circ}$ edição. São Bernardo do Campo, 2014. Disponível em: http://www. saobernardo.sp.gov.br/documents/10181/26510/ss_osteoporose.pdf/c8fec424-7990-48f9-87c9-59899d7217d6.

6. Ministério da Saúde (BR). Resolução № 466, de 12 de Dezembro de 2012. Brasília (DF). Diário Oficial da União. Disponível em: http://bvsms.saude.gov.br/bvs/saudelegis/cns/2013/res0466_12_12_2012.html. Acesso em: 10 nov. 2017.

7. Abrahin O, Rodrigues RP, Marçal AC, Alves EAC, Figueiredo RC, Sousa EC. Natação e ciclismo não causam efeitos positivos na densidade mineral óssea: uma revisão sistemática. São Paulo. Rev. Bras. Reumatol., 2016, 56(4): 345-351. DOI: http:// dx.doi.org/10.1016/j.rbre.2016.02.013.

8. Silva $A C V$, Rosa MI, Fernandes $B$, Lumertz S, Diniz RM, Damiani MEFR. Fatores associados à osteopenia e osteoporose em mulheres submetidas à densitometria óssea. São Paulo. Rev. Bras. Reumatol., 2015, 55(3): 223-228. DOI: http://dx.doi. org/10.1016/j.rbr.2014.08.012.

9. Garcia PA, Dias JMD, Rocha ASS, Almeida NC, Macedo OG, Dias RC. Relação da capacidade funcional, força e massa muscular de idosas com osteopenia e osteoporose. São Paulo. Fisioter. Pesqui., 2015, 22(2): 126-132. DOI: http://dx.doi. org/10.590/1809-2950/13154522022015. 
10. Oliveira LG, Guimarães MLR. Osteoporose no homem. São Paulo. Rev Bras Ortop. 2010; 45(5): 392-6. DOI: http://dx.doi. org/10.1590/S0102-36162010000500003.

11. Anvisa. Agência Nacional de Vigilância Sanitária. Saúde e Economia - Osteoporose. Ano I - Edição n 1 agosto, 2009. Disponível em: http://portal.anvisa.gov.br/documents/33884/412285/Boletim+Sa\%C3\%BAde+e+Economia+n\%C2\%BA+ 1/7d49dea4-6610-4a71-9154-c4df75c3dd53.

12. Ministério da Saúde (BR). Protocolo Clínico e Diretrizes Terapêuticas Osteoporose. Portaria Secretaria de Atenção à Saúde/ Ministério da Saúde n 451, de 9 de junho de 2014. Disponível em: http://portalsaude.saude.gov.br/images/pdf/2014/ dezembro/15/Osteoporose.pdf.

13. Pereira RDM, Alvim NAT, Pereira CD, Gomes Junior, SC. Acupuntura na hipertensão arterial sistêmica e suas contribuições sobre diagnósticos de enfermagem. Rio de Janeiro. Esc. Anna Nery, 2017, 21(1). DOI: http://dx.doi.org/10.5935/14148145.20170024 .

14. Porfirio E, Fanaro GB. Suplementação com colágeno como terapia complementar na prevenção e tratamento de osteoporose e osteoartrite: uma revisão sistemática. Rio de Janeiro. Rev. bras. geriatr. gerontol., 2016, 19.(1):153-164. DOI: http://dx.doi. org/10.1590/1809-9823.2016.14145.

15. Souza SPMC, Morais FE, Santos EV, Silva ML, Martinez-Huitle CA, Fernandes NS. Determinação do teor de cálcio em comprimido à base de lactato de cálcio utilizado no tratamento da osteoporose. São Paulo. Quím. Nova, 2012, 35(7): 13551359. DOI: http://dx.doi.org/10.1590/S0100-40422012000700013.

16. Bellan M, Pirisi M, Sainaghi PP. Osteoporose na artrite reumatoide: papel do sistema vitamina D/hormônio paratireóideo. São Paulo. Rev. Bras. Reumatol. 2015, 55(3): 256-263. DOI: http://dx.doi.org/10.1016/j.rbr.2014.10.007.

17. Khajuria DK, Razdan R, Mahapatra DR. Medicamentos para o tratamento da osteoporose: revisão. São Paulo. Rev Bras Reumatol., 2011, 51(4): 365-82. DOI: http://dx.doi.org/10.1590/S0482-50042011000400008.

18. Pinheiro MM, Szejnfeld VL. Tratamento da osteoporose. Sinopse de Reumatologia. São Paulo. Moreira Jr Editora, 2004, 6(4): 102-121. Disponível em: http://www.moreiraj.com.br/revistas.asp?id_materia=2773\&fase=imprime. Acesso em: 28 set. 2016.

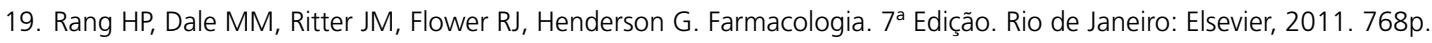

20. Maeda SS, Borba VZC, Camargo MBR, Silva DMW, Borges JLC, Bandeira F, Lazaretti-Castro M. Recomendações da Sociedade Brasileira de Endocrinologia e Metabologia (SBEM) para o diagnóstico e tratamento da hipovitaminose D. São Paulo. Arq Bras Endocrinol Metab., 2014, 58(5), DOI: http://dx.doi.org/10.1590/0004-2730000003388.

21. Stolnicki B, Oliveira LG. Para que a primeira fratura seja a última. São Paulo. Rev. bras. ortop., 2016, 51(2): 121-126. DOI: http://dx.doi.org/10.1016/j.rboe.2016.01.005.

22. Driusso P, Neves VFC, Granito RN, Rennó ACM, Oishi J. Redução da dor em mulheres com osteoporose submetidas a um programa de atividade física. São Paulo. Fisioter. Pesq., 2008, 15(3): 254-8. DOI: http://dx.doi.org/10.1590/S180929502008000300007.

23. Ares MJJ. Utilização de órteses na reabilitação de pacientes portadores de osteoporose vertebral. São Paulo. Acta Fisiátrica, 1997, 4(2)(supl.1): 148-152.

24. Soares DS, Mello LM, Silva AS, Nunes AA. Análise dos fatores associados a quedas com fratura de fêmur em idosos: um estudo caso-controle. Rio de Janeiro. Rev. Bras. Geriatr. Gerontol., 2015, 18(2): 239-248. DOl: http://dx.doi.org/10.1590/18099823.2015.14022. 
25. Ely LS, Engroff P, Guiselli SR, Cardoso GC, Morrone FB, Carli GA. Uso de anti-inflamatórios e analgésicos por uma população de idosos atendida na Estratégia Saúde da Família. Rio de Janeiro. Rev. bras. geriatr. gerontol., 2015, 18(3): 475-485. DOI: http://dx.doi.org/10.1590/1809-9823.2015.14141.

26. Sarinho ESC, Melo VMPP. Doença Óssea Induzida pelos Glicocorticoides: Mecanismos e Importância na Prática Pediátrica. São Paulo. Rev. paul. pediatr. 2017, 35(2): 207-215. DOI: http://dx.doi.org/10.1590/1984-0462;2017/;35;2;00007.

27. Manolagas SC, Weinstein RS. New developments in the pathogenesis and treatment of steroid-induced osteoporosis. Estados Unidos. J. Bone Miner. Res., 1999, 14(7): 1061-1066. DOI: http://dx.doi.org/10.1359/jbmr.1999.14.7.1061.

28. Assugeni R, Parentoni RB, Abreu R, Destri C. Manual do paciente. Instituto EnzoAssugeni, Copyright 2007. Disponível em: http://www.sincomercioata.com.br/Legislacoes/Arquivos/Manual_Paciente.pdf.

29. Camargo EP, Minosso M, Lopes LC. Caracterização do uso de alendronato de sódio no tratamento de osteoporose por clínicos da rede privada de duas cidades do interior de São Paulo. São Paulo: Araraquara. Rev. Ciênc. Farm. Básica Apl., 2007, 28(1): 77-83.

30. Brasil. Manual das Denominações Comuns Brasileiras - MDCB, coordenadores Lauro D. Moretto, Rosana Mastelaro. São Paulo: Sindusfarma, 16, 2013. Disponível em: http://www.anvisa.gov.br/hotsite/farmacopeiabrasileira/conteudo/2013/ Manual\%20DCB\%202013\%20Vers\%C3\%A30\%20final.pdf.

31. Santos V, Nitrini SMOO. Indicadores do uso de Medicamentos Prescritos e de Assistência ao Paciente de Serviços de Saúde. São Paulo. Ver. Saúde Pública, 2004, 38(6): 819-26. DOI: http://dx.doi.org/10.1590/S0034-89102004000600010.

32. Ferreira MBC, Heineck I, Flores LM, Camargo AL, Dal Pizzol TS, Torres ILS, Koenig A, Trevisol DJ, Melo AMMF, Cardoso TFM, Monreal MTFD, Kadri MCT. Rational use of medicines: prescribing indicators at different levels of health care. São Paulo. Braz. J. Pharm. Sci., 2013, 49(2): 329-340. DOI: http://dx.doi.org/10.1590/S1984-82502013000200015.

33. Inbravisa - Instituto Brasileiro de Auditoria em Vigilância Sanitária. Manual Prático para Prescrição de Medicamentos de acordo com a legislação sanitária brasileira, 2010. Disponivel em: http://www.sbrafh.org.br/site/public/temp/5161 ea3ccde67.pdf.

34. Madruga CMD. Manual de orientações básicas para prescrição médica. $2^{a}$ edição. Rev. Ampl., Brasília: CRM-PB/CFM, 62p, 2011. Disponível em: http://portal.cfm.org.br/images/stories/biblioteca/cartilhaprescrimed2012.pdf. 